\title{
Solitary fibrous mediastinal tumor with coronary vascular supply: An unusual case
}

\author{
N. Qedra, MD, M. Kadry, MD, PhD, E. Ivanitskaia-Kühn, MD, S. Buz, MD, R. Meyer, MD, PhD, \\ H. Laube, MD, PhD, and R. Hetzer, MD, PhD, Berlin, Germany
}

Solitary fibrous tumor (SFT) of the mediastinum occurs most commonly in the visceral and parietal pleura; it is extremely rare for it to affect cardiac structures. We report, for the first time, a case of mediastinal SFT with an unusual vascular supply from the coronary arteries and an unusual location. The tumor was diagnosed 34 months after aortic valve replacement and revascularization of the left anterior descending coronary artery with the left internal thoracic artery (ITA). It was completely resected, and 16 months later, there have been no local or distant recurrences.

\section{CLINICAL SUMMARY}

A 68-year-old man underwent aortic valve replacement, reduction plasty of the ascending aorta, and revascularization of the left anterior descending coronary artery with the left ITA. The perioperative chest radiograph was without pathologic findings (Figure 1, A). Because of planned inguinal hernia repair 34 months later, a routine chest roentgenogram was taken, and this showed a well-delineated abnormal shadow (Figure 1, $B$ ). At this time, the patient was asymptomatic. At an external hospital, sonography-guided needle aspiration of the mass yielded suspected SFT, and the patient was transferred to our institute for surgical resection.

Preoperative coronary angiographic analysis showed the tumor to be supplied by the conus artery originating from the right coronary artery and by the sinus node artery originating from the right circumflex artery (Figure 1, $C$ and $D$ ).

Computed tomographic (CT) scanning demonstrated a well-circumscribed mass measuring $10.05 \times 5 \times 9 \mathrm{~cm}$ (mean density, -51.5 Hounsfield units) directly behind the sternum and extending to the ascending aorta, the right atrium, and the right ventricle with contact to the right coronary artery (Figure 2, $A$ and $B$ ).

Access to the mass was accomplished through a median sternotomy. Because of the adhesions, no tumor capsule

\footnotetext{
From the Department of Cardiothoracic and Vascular Surgery, Deutsches Herzzentrum Berlin, Berlin, Germany.

Disclosures: None.

Received for publication Aug 1, 2008; accepted for publication Aug 21, 2008; available ahead of print Jan 19, 2009.

Address for reprints: Naser Qedra, MD, Deutsches Herzzentrum Berlin, Augustenburger Platz 1, 13353 Berlin, Germany (E-mail: qedra@gmx.de).

J Thorac Cardiovasc Surg 2010;139:e23-5

0022-5223/\$36.00

Crown Copyright (c) 2010 by The American Association for Thoracic Surgery doi:10.1016/j.jtcvs.2008.08.038
}

was identified. After preparation of the heart, protecting the ITA bypass, the right pleura was opened, and the firm, fist-sized, whitish mass was demarcated. The tumor was completely resected without extracorporeal circulation. The postoperative course was uneventful.

The resected tumor, consisting of a $12.5 \times 9.5 \times 4.7-\mathrm{cm}$ $(258 \mathrm{~g})$ mass, showed no necrosis or calcification. Microscopic examination showed a mesenchymal tumor formed predominantly of collagen fibers. The tumor cells displayed relatively little pleomorphism, with very rare mitoses. Results of immunohistochemical staining were positive for vimentin, collagen-1, and collagen-3 and negative for desmin, pancytokeratin, anti-cytokeratin monoclonal antibodies (AE1 and AE3), and collagen-2. Results of Ki-67 staining were negative. A final diagnosis of a benign, cell-poor fibrous tumor was made (Figure 2, C).

Sixteen months later, the patient is in good health, and CT scans of the thorax and abdomen have revealed no signs of local or distant tumor recurrence (Figure 2, D).

\section{DISCUSSION}

Most pleural SFTs are located in or arise from the visceral $(80 \%)$ and parietal $(20 \%)$ pleura, with an incidence of 2.8 cases per 100,000. Approximately 800 cases have been reported in the literature thus far. ${ }^{1}$ After revision of the pleural tumor classification by the World Health Organization in 1999, pleural SFT is now classified as an independent entity and excluded from the mesothelioma subgroups.

At present, only 7 cases of mediastinal SFT originating from the pericardium have been described in the literature. El-Naggar and colleagues ${ }^{2}$ reported in 1989, for the first time, the histology of a case of intrapericardial SFT, followed by Bortolotti's report of an intrapericardial SFT attached only to the ascending aorta and pulmonary trunk. ${ }^{3}$ In a third case presented by Flemming and coworkers, ${ }^{4}$ the tumor was on the left ventricular side, involving the epicardium but with no invasion of the myocardium. In 3 other cases of pericardial SFTs reported by Corgnati, Segawa, and Val-Bernal, the tumor had infiltrated the right ventricle. ${ }^{3}$ In a case presented by Andreani and associates, ${ }^{5}$ a tumor in the anterosuperior mediastinum displaced the trachea, large vessels, and esophagus.

In our case the tumor's close vicinity to the right ventricle led us to suspect a pericardial origin, although a pleural origin cannot be excluded because of the adhesions and the open pericardium. Two factors make our case worthy of presentation: the unusual supply of the tumor by the 

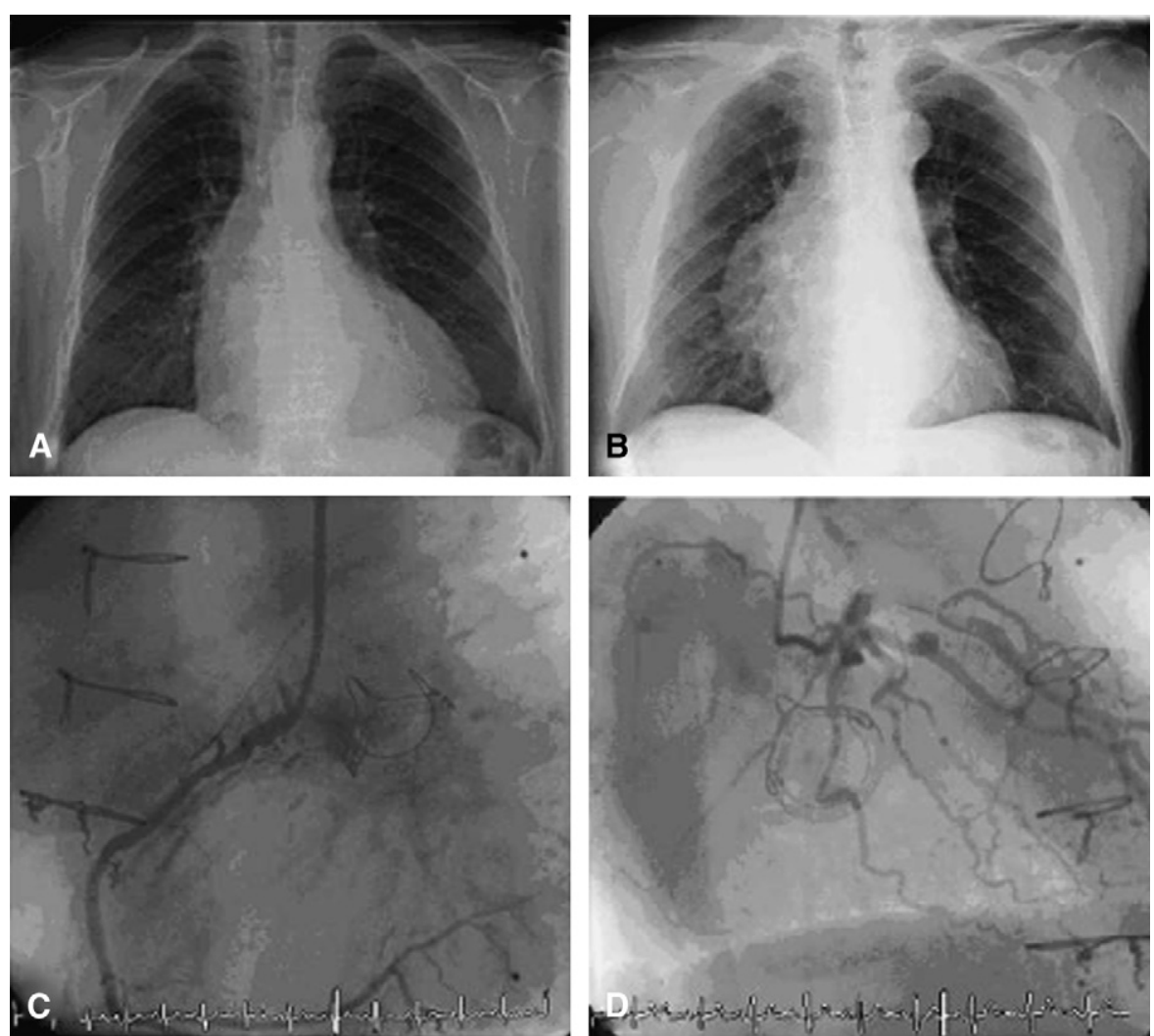

FIGURE 1. Roentgenogram before aortic valve replacement (A) and 34 months later (B) and coronary angiogram showing the tumor supply by the conus artery originating from the right coronary artery $(C)$ and by the sinus node artery originating from the right circumflex artery (D).
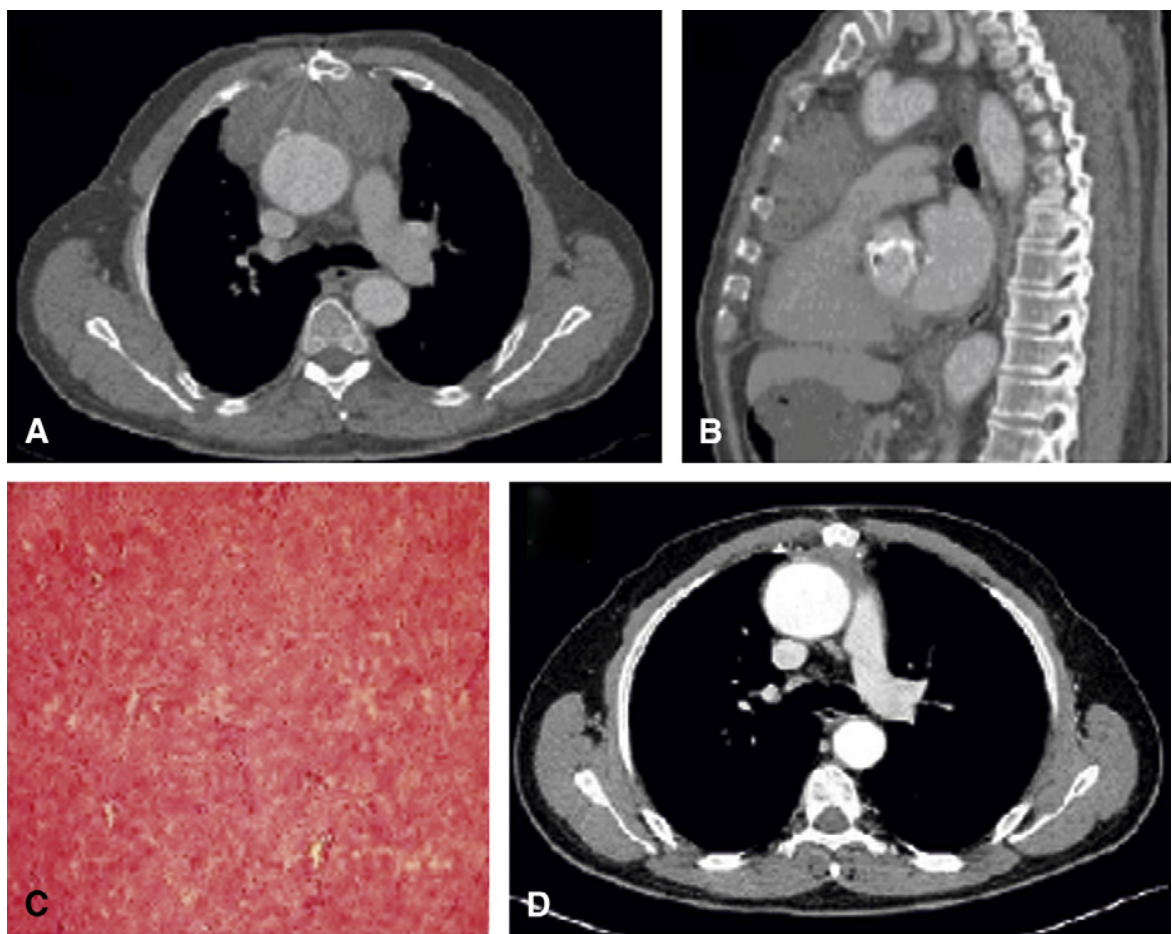

FIGURE 2. A and B, Computed tomographic scan demonstrating the extent of the tumor mass in contact with the heart and great vessels in the transverse (A) and sagittal (B) axis. C, The histology of the tumor mass. (Hematoxylin and eosin stain, original magnification $\times 100$ ). D, Computed tomographic scan of the chest 16 months after tumor resection. 
coronary artery and its relatively rapid growth as a fibroma. The growth period might have been even less than 34 months because no imaging was undertaken during the postoperative period after aortic valve replacement.

The preoperative CT scan and angiogram were of great help in planning complete resection of the tumor, avoiding injury to the coronary arteries and the left ITA under reoperation conditions and without extracorporeal circulation.

Because local recurrence is possible in benign, as well as malignant, forms of SFT resected incompletely, radical resection should be strived for in all patients.

\section{References}

1. De Perrot M, Fischer S, Bründler MA, Sekine Y, Keshavjee S. Solitary fibrous tumors of the pleura. Ann Thorac Surg. 2002;74:285-93.

2. El-Naggar AK, Ro JY, Ayala AG, Ward R, Ordóñez NG. Localized fibrous tumor of the serosal cavities. Immunohistochemical, electron-microscopic, and flow-cytometric DNA study. Am J Clin Pathol. 1989;92:561-5.

3. Val-Bernal JF, Figols J, Gómez-Román JJ. Incidental localized (solitary) epithelial mesothelioma of the pericardium: case report and literature review. Cardiovasc Pathol. 2002;11:181-5.

4. Flemming P, Maschek H, Werner M, Kreft A, Graeter T, Georgii A. Solitary fibrous tumor of the epicardium. Pathologe. 1996;17:139-44.

5. Andreani SM, Tavecchio L, Giardini R, Bedini AV. Extrapericardial solitary fibrous tumour of the pericardium. Eur J Cardiothorac Surg. 1998; 14:98-100

\title{
A word of caution: Cerebral air emboli caused by tubing elastic recoil while performing low-flow antegrade cerebral perfusion in a low-birth-weight neonate
}

\author{
Georgy Frenkel, MD, ${ }^{\mathrm{a}}$ Einat Birk, MD, ${ }_{\mathrm{b}}$ Bernardo Vidne, MD, ${ }^{\mathrm{a}}$ Golan Shukrun, PCP, ${ }^{\mathrm{a}}$ Oren Bachar, PCP, ${ }^{\mathrm{a}}$ \\ Yakov Katz, MD, ${ }^{\mathrm{c}}$ and Gabriel Amir, MD, PhD, ${ }^{\mathrm{a}}$ Petach Tikva, Israel
}

With continuous improvement of cardiac surgical techniques, we are currently able to perform complex congenital heart surgery, even in small neonates. ${ }^{1,2}$ Some of the complex operations of the aortic arch involve hypothermia and either deep hypothermic circulatory arrest or antegrade cerebral perfusion (ACP). Traditionally, deep hypothermic circulatory arrest has been used when neonatal arch surgery was performed. Recently, the use of ACP has been advocated as a means of brain protection. ${ }^{3}$ Two basic techniques for ACP have been used: either suturing a polytetrafluoroethylene shunt (Gore-Tex shunt; W. L. Gore \& Associates, Inc, Flagstaff, Ariz) to the innominate artery or direct cannulation of either the ascending aorta or the innominate artery. We herein report a case in which ACP was used in a 2-kg neonate. Once ACP was initiated, air bubbles were seen in the arterial cannula, rising toward the pump. The air bubbles

\footnotetext{
From the Division of Pediatric Heart Surgery, ${ }^{\mathrm{a}}$ Department of Pediatric Cardiology, and Pediatric Anesthesia, ${ }^{\mathrm{c}}$ Schneider Children's Medical Center of Israel, Petach Tikva, Israel.

Disclosures: None.

Received for publication March 27, 2008; revisions received Aug 17, 2008; accepted for publication Aug 27, 2008; available ahead of print Jan 19, 2009.

Address for reprints: Gabriel Amir, MD, PhD, Division of Pediatric Heart Surgery, Schneider Children's Medical Center of Israel, 14 Kaplan St, Petach Tikva 49202, Israel (E-mail: gabrielA@clalit.org.il).

J Thorac Cardiovasc Surg 2010;139:e25-6 0022-5223/\$36.00

Crown Copyright (C) 2010 Published by Elsevier Inc. on behalf of The American Association for Thoracic Surgery

doi:10.1016/j.jtcvs.2008.08.037
}

were caused by a tubing recoil phenomenon apparent only when low pump flows were used.

\section{CLINICAL SUMMARY}

A premature 32-week-old neonate was given a diagnosis of severe coarctation and posterior malalignment ventricular septal defect. Arch anatomy consisted of a left aortic arch with an aberrant right subclavian artery. The operation was performed through a median sternotomy. After subtotal thymectomy and opening of the pericardium, cardiopulmonary bypass (CPB) was initiated through a 3.5 -mm polytetrafluoroethylene shunt sutured to the right carotid artery, the aortic cannula was connected to the shunt, and venous return was obtained through bicaval venous cannulation. Pump occlusion was checked before the initiation of CPB. CPB was initiated, and the patient was cooled to $18^{\circ} \mathrm{C}$. the aortic arch was dissected, and the anatomy was evaluated; the ductus arteriosum was ligated and divided. Snares were placed around the left and right carotid arteries and the left subclavian artery. The right subclavian artery was incorporated in the distal clamp. Once the temperature reached $18^{\circ} \mathrm{C}$, the brachiocephalic vessels were clamped, and ACP was initiated $\left(30 \mathrm{~mL} \cdot \mathrm{kg}^{-1} \cdot \mathrm{min}^{-1}, 60 \mathrm{~mL} / \mathrm{min}\right)$. Arch reconstruction was initiated. On initiation of ACP, air bubbles were seen in the arterial cannula flowing toward the pump. Perfusion was immediately stopped, and protective measures were undertaken. The patient was positioned in a deep Trendelenburg position, and retrograde brain perfusion was initiated through the superior vena cava. Aortic arch 